\title{
Imaging salivary glands post external radiation beam therapy of head and neck
}

\section{tumours}

\author{
Mpumelelo Nyathi
}

Department of Medical Physics, School of Medicine, Sefako Makgatho Health Sciences University,

\section{Ga-Rankuwa, South Africa}

\section{REVIEW}

Please cite this paper as: Nyathi M. Imaging salivary glands post external radiation beam therapy of head and neck tumours. AMJ 2018;11(6):335-342.

https://doi.org/10.21767/AMJ.2018.3412

\section{Corresponding Author:}

Mpumelelo Nyathi

Department of Medical Physics, School of Medicine

Sefako Makgatho Health Sciences University

Box 672, Medunsa, Ga-Rankuwa, South Africa

Email: Mpumelelo.Nyathi@smu.ac.za

\section{ABSTRACT}

\section{Background}

Radiation therapy using external radiation beam is an established treatment for head and neck cancers. However, salivary glands are exposed leading to severe impairment of oral health. In order to devise patient care strategies, clinicians can measure the degree of salivary gland impairment using various imaging modalities.

\section{Aims}

The study aimed determining the best imaging modality for measuring salivary glands impairment post external radiation beam therapy of head and neck tumours.

\section{Methods}

A systematic literature review, performed using Google and Science Direct.com search engines. Publications in English on salivary gland imaging modalities post external radiation beam therapy of head and neck cancers were selected. Information on advantages and disadvantages of the imaging modalities was documented.
Results

The study established that the Magnetic Resonance Imaging (MRI), Computer Tomography (CT), Ultra Sound (US) depict structural changes while salivary gland scintigraphy provides functional information. It was also established that the obstruction of the salivary glands can be measured using Magnetic Resonance - Sialography.

\section{Conclusion}

The salivary gland scintigraphy technique is the most sensitive of all salivary gland imaging modalities with the ability to detect slight parenchymal injuries well before structural changes can be seen using the MRI, CT and US. However, when evaluating the obstruction of the salivary glands ducts, the Magnetic Resonance-Sialography modality offers the best option.

\section{Key Words}

Salivary glands, imaging modalities, head and neck tumours, external radiation beam therapy

\section{What this review adds:}

\section{What is known about this subject?}

During external beam radiation therapy of head and neck tumours, salivary glands are impaired leading to oral complications among patients.

\section{What new information is offered in this review?}

Salivary glands scintigraphy detects the slightest changes in the function of the salivary glands well before structural changes can be detected using the MRI, the CT and US.

\section{What are the implications for research, policy, or} practice?

Oncologist and clinicians should subject patients treated for head and neck patients to salivary gland scintigraphy during and post treatment in order to assess salivary glands' damage. Information acquired would help to optimize therapy as well as to come up with individualised patient care strategies that would alleviate suffering arising from 
the side effects of therapy.

\section{Introduction}

Radiation therapy is an established modality for treatment of head and neck cancers. ${ }^{1,2}$ However, it is known to cause salivary glands impairment resulting in dysfunction and xerostomia. ${ }^{1}$ Salivary glands impairment can be reduced through use of modern radiation techniques such as the three dimensional conformal radiation and the intense modulated therapy (IMRT). ${ }^{2,3}$ However, it is not always economically feasible for all patients to be treated using the modern techniques owing to their economic status hence the use of techniques that do not spare radiation intensity on the healthy tissue in the head and neck region. Unintentionally irradiation of the salivary glands results in hypofunction leading to reduced saliva flow rates and numerous oral complications for patients treated for head and neck cancers. In order to optimize treatment, oncologists need to understand the side effects of therapy. This can only be possible through imaging the salivary glands during and post therapy. This review was aimed at selecting the best imaging modality for extracting information on the degree of injury of the salivary glands post external beam radiation therapy of head and neck tumours.

The article firstly gives the reader an overview of head and neck cancers, proceeding to briefly discuss salivary glands and the role of saliva. Finally existent salivary glands imaging modalities are examined with focus on their strengths and limitations when evaluating salivary glands injuries during and post external beam radiation therapy of head and neck tumours.

\section{Head and neck tumours}

Head and neck tumours occur in various parts of the anatomical region of the head and neck. They include tumours of the salivary glands, larynx, nasal cavities, middle ear and lymph nodes in the upper neck. Among the wellestablished risk factors are use of tobacco and alcohol. However, current evidence link infection by papillomavirus as a risk factor of nasopharyngeal carcinoma and laryngeal carcinoma. ${ }^{4}$ Typical symptoms include a lump or sore throat that does not resolve, difficulties in swallowing and a change or hoarseness of the voice. ${ }^{4,5}$

Incidences of head and neck tumours appear to be on the rise. As many as 50,000 new cases have been registered annually in USA. ${ }^{4,6}$ Asian countries have not been spared either. In China and India, the head and neck tumours are listed among the most common types of cancer. However, in most developing countries particularly in Africa, the magnitude of head and neck tumour incidences is difficult to appreciate since many countries do not keep cancer registers. $^{7-9}$ On a lighter note, it can be said that the perceived rise in head and neck incidences can be attributed to the advances in diagnostic technologies and people living much longer particularly in developing countries.

\section{Salivary glands}

The salivary glands are classified into two categories, the major and the minor salivary glands. The major glands are paired. ${ }^{10,11}$ These include the parotid salivary glands (PSGs), the submandibular salivary glands (SMGs) and the sublingual salivary glands (SLGs) listed in the order of decreasing size. ${ }^{10}$ The minor salivary glands may be found on the lips, in the inner cheek area and throughout the pharynx. $^{10,12}$

The PSGs are made of serous cellular cells which produces watery, ${ }^{13}$ and serous secretions. ${ }^{11,13}$ The serous type of acini is made up of a homogeneous population of the polarized cells that have abundant granular cytoplasm. ${ }^{13}$ In infants, the acini constitute of mucous cells. However, these cells become serous at adulthood. The human parotid gland acini consist of the pyramedial secretary cells and flattened basally placed myoepithelial cells. They are considered to be seromucous glands. The secretary cells show a cytological organization intermediate to exocrine pancreas and that of the mucous glands. ${ }^{14,15}$ The PSGs produce more than 50 per cent of saliva when stimulated and 28 per cent when unstimulated. ${ }^{11}$ The submandibular salivary glands (SMGs) consist of mainly acini that comprises of exclusively mucous cells, a mixture of serous and mucous cells. The serous and mucous acini cells differ in their histochemical properties hence differences in their biochemical properties and secretions. ${ }^{14}$ The SMGs produce mixed saliva whereas the SLGs produced mucous saliva. Both the SMGs and the SLGs produce 68 per cent of unstimulated saliva. The minor salivary glands produce only a small proportion of mucous saliva. $^{11}$

\section{Functions of the saliva}

The saliva secreted by the acini apparatus is controlled by the autonomic nervous system. Saliva acts as a lubricant for all the food taken in the mouth. It binds the masticated food into a bolus making it to pass easily through the gullet without injuring the mucosa. ${ }^{14,15}$ Furthermore, the saliva moistens the oral cavity thereby precluding the ill-effects such as inflammation, ulceration and general discomfort. Saliva contains lysozyme which kills bacteria in the oral cavity and it provides a buffering alkaline medium. The 
serous acinar cells produces saliva together with an enzyme called salivary amylase. This enzyme initiates the digestion of starch. ${ }^{11,16-19}$ Reduction in saliva flow rates may be prompted by radiation injury of the salivary glands, causing xerostomia. $^{19,20}$ Lastly, saliva, brings to the enamel phosphates, fluoride and peptides which play a significant role in remineralization. ${ }^{11}$

\section{Radiation induced Xerostomia}

Radiation-induced xerostomia is a well-publicised sideeffect of radiation therapy of head and neck tumours. ${ }^{21,22}$ However, it is dose dependent. The salivary glands can tolerate doses from $32 \mathrm{~Gy}$ to $46 \mathrm{~Gy}$, beyond which dysfunction may be permanent resulting in xerostomia. Generally curative external radiation beam therapy doses may be up to 60Gy, hence injury of salivary glands leading to formation of xerostomia, ${ }^{23}$ which can either be chronic or acute. ${ }^{24}$ Acute xerostomia is characterised by oral mucositis, fungal, and bacterial infection, sialadentis and taste dysfunction while chronic xerostomia is characterised by soft-tissue necrosis and dental caries. ${ }^{24}$

\section{Medical imaging}

McGraw-Hill Dictionary of Scientific \& Technical Terms defines medical imaging as "the production of visual representations of body parts, tissues, or organs, for use in clinical diagnosis; encompasses $\mathrm{X}$-ray methods, magnetic resonance imaging, single-photon-emission and positronemission tomography, and ultrasound". ${ }^{25}$ Medical imaging can facilitate acquisition of salivary glands images that can be used to determine the degree of their malfunction with view of optimization of treatment of patients treated for head and neck cancers.

Salivary glands are amenable to imaging with Magnetic Resonance Imaging (MRI), Computed Tomography (CT), Ultrasound (US), Sialography, Magnetic Resonance Sialography (MR-Sialography) and scintigraphy modalities. ${ }^{26-}$

33 Imaging salivary glands post-radiation therapy has become a necessity. Images of the salivary glands can be used to evaluate the side effects of radiation therapy as they can provide the clinicians with crucial information on the extent of their malfunction. The information is crucial in devising patient care strategies aimed at alleviating suffering caused by complications arising from therapy, in particular xerostomia.

Xerostomia affects majority of people treated using external radiation beam therapy for oropharyngeal, hypopharyngeal, laryngeal, and nasopharyngeal carcinomas, either at onset of treatment or post treatment. ${ }^{3}$ Several studies have been conducted about xerostomia. ${ }^{20-23}$ However, despite the hindrance impact of xerostomia, clinician have mainly focused on treatment of head and neck tumours. Less emphasis has been focused on determining the best imaging modality for imaging salivary glands with view of measuring their degree of dysfunction post external radiation beam therapy of the head and neck tumours. The current study was therefore aimed at determining the best imaging modality measuring the function of the salivary glands post radiation therapy of the head and neck tumours.

\section{Methods}

A systematic literature review was performed using Google search engine and Science Direct.com for publications in English on salivary gland imaging modalities. Publications were screened to retrieve information on the salivary gland imaging modalities, their advantages and disadvantages when used to image salivary glands post external radiation beam therapy of head and neck tumours. The articles were searched using the keywords; salivary glands imaging postradiation therapy, salivary glands function and head and neck tumours, radiation xerostomia, computed tomography and salivary glands, magnetic resonance imaging and salivary glands, ultrasound imaging and salivary glands, salivary glands scintigraphy and Magnetic ResonanceSialography modality. Sources were limited to articles dealing with external beam radiation therapy, salivary gland imaging post external radiation beam therapy, parotid salivary glands, head and neck carcinomas. Supplementary references were identified from the references identified in the data bases consulted. Figure 1 shows the article search steps. The study was approved by the Medunsa Research Ethics Committee under the number MREC/P/280/2012: PG.

\section{External Beam Radiation Therapy of head and neck cancers and consequences}

External beam radiation therapy for head and neck cancers plays a significant role in the control of loco-regional disease and improved survival rates. However, use of ionizing radiation in the head and neck region leads to both acute and long terms complications arising from injury of healthy tissue mainly the salivary glands. Irradiation of the salivary glands leads to necrosis of acinar cells resulting in alteration of their volume. ${ }^{21}$

The impact of ionizing radiation on salivary glands takes place in phases. During the early phase, acute swelling of the salivary glands occurs caused by either duct obstruction or interstitial oedema or both. A month after treatment, 
massive degeneration of the salivary glands has been reported and this continues to the third month after treatment. Thereafter, four months plus, patch distortions of nearly all acini are observable and few serous cells remain while the ducts and lobules undergo fibrosis. ${ }^{34}$ Atri et al., ${ }^{22}$ observed that the salivary glands can tolerate ionizing radiation dose in the range of $32 \mathrm{~Gy}$ to $46 \mathrm{~Gy}$. However, curative doses may range up to 60Gy and sometimes more. Irradiation of salivary glands with high doses may lead to salivary glands dysfunction accompanied by severe side effects that compromise the quality of life of patients treated for head and neck carcinoma. ${ }^{22}$

Although it has long been established that radiation therapy of head and neck cancers causes salivary glands destruction and dysfunction leading to radiation induced xerostomia, $3,21-$ ${ }^{23}$ there still remains a need for establishment of a suitable procedure of quantifying the degree of injury of the salivary glands. Medical imaging provides us with a suitable procedure because salivary glands are responsive to imaging using $\mathrm{MRI}, \mathrm{CT}$, US and radionuclide imaging. ${ }^{26-34}$ However, it is important that the best imaging modality be determined.

\section{Magnetic Resonance Imaging}

Magnetic Resonance Imaging is a unique and exciting medical imaging technique. It is associated with the phenomenon of nuclear magnetic resonance (NMR). MRI scanners are similar to $\mathrm{CT}$ scanners but their imaging principles differ. Unlike CT, MRI does not use ionising radiation to produce images. The MRI imaging signal is dependent on the radio wave communication with a patient placed within a magnetic field. It has the capacity to detect slight changes taking place in the nucleus of an atom. ${ }^{35} \mathrm{MRI}$ images can clearly differentiate between healthy tissues and soft tissue lesions compared to any imaging modality. ${ }^{36}$ Furthermore, the MRI modality has the ability to clearly differentiate between adjacent anatomic structures. ${ }^{36}$ The MRI modality offers good spatial resolution and contrast resolution. $^{33}$

Some researchers have advocated the use of MRI when imaging the salivary gland ductal system based on its ability to offer weighted images. ${ }^{36,37}$ The parotid salivary glands that have been exposed to radiotherapy of 60-70 Gy when imaged by MRI system will show a pronounced reduction in volume. $^{36}$ A similar reduction in volume has also been observed on imaging submandibular salivary glands. The decrease in the volume of the salivary glands after radiotherapy is associated with loss of acinar cells. ${ }^{36,37}$ The MRI has the ability to visualise deep located structures such as the parotid salivary gland lobe hence can be used to evaluate swelling of the parotid salivary glands. ${ }^{36}$ The fatty background around parotid salivary glands makes it possible to clearly visualise any abnormalities using MRI with weighted images. ${ }^{37}$

Despite all the glamour and the high resolution offered by MRI images, the modality has some noticeable disadvantages as a salivary gland imaging modality. It is very expensive, ${ }^{33}$ and thus cannot be used routinely to monitor salivary glands function during external beam radiation therapy of head and neck tumours particularly in developing countries. Furthermore, the MRI modality is a contra indication for patients with metallic implants and other nonMRI compatible surgical clips. ${ }^{36,37}$ Patients who are claustrophobic have been reported to have difficulties in tolerating the confined MRI space as well those who are afraid of noise can hardly tolerate MRI. ${ }^{36}$

\section{Computed Tomography}

The $\mathrm{CT}$ is an imaging technique that is used to acquire digital images. $^{36,37}$ It can acquire the images in three planes namely; coronal, sagittal and axial. ${ }^{31}$ Furthermore, it has the ability to reconstruct cross-sectional images of the body from X-ray projections acquired at various angles around the body or organ producing three dimensional (3D) images. $^{36}$ It has since been established as one of the techniques for evaluating salivary glands sailodiantes. ${ }^{36,37}$ However, there is little information in literature about its role as an assessment modality for post radiation salivary glands. The success of the CT as an imaging modality can be attributed to its ability to directly visualise soft tissue and hard tissues. ${ }^{36}$ It has been successfully used for evaluating the deep parotid gland lobe where MRI modality could not reach. It provides a good view of intraglandular lobes. ${ }^{36,37}$ It can easily show the whole physical volume of the parotid gland in the presence of an iodinated contrast medium which makes the parotid ducts visible.

The CT modality offers sequences of data or information in the form of thin slices. The information in a selected slice does not suffer from interference of structures that are external to the slice. The slice gives an opportunity to study and analyse the nature of matter which is inert, static or even dynamic. Furthermore, it makes it easy to understand the features and characteristics of this matter. ${ }^{36}$ The ability of the CT modality to offer a 3D view of the targeted organ makes it a resourceful imaging modality for study of the anatomical structure and morphological changes of the salivary glands post-radiation therapy. Another main advantage of this modality is its abilities to eliminate the 
superimposition of anatomical structures thereby giving an unobstructed view of the organ under study.

Cheng et al., ${ }^{36}$ conducted a study on morphological changes of salivary glands post radiation therapy. They found out that the parotid salivary glands and the submandibular salivary glands showed increased image density post radiation therapy. They attributed this difference to the presence of the contrast medium stored in the spaced previously occupied by the acinar cells. However, the increased image density of salivary glands occurs when doses used are greater than $45 \mathrm{~Gy}$ yet salivary glands can be injured at lower doses. This makes the sensitivity of the CT modality a cause of concern. Furthermore, increased image density has also been reported in CT images of patients with salivary gland tumours. In this light image density should not be associated at first hand with radiation inflicted injuries as it might be due to other underlying factors. Spray artefact from dental filling also limit use of the CT modality for evaluating salivary gland function post radiation treatment. Lastly patients with renal failure cannot have their salivary glands evaluated with the CT modality because its uses a contrast medium. ${ }^{34,36}$

\section{Ultra sound}

The US is a diagnostic modality used to obtain morphological information about a biological medium. It acquires tomography slices without use of ionising radiation, ${ }^{27,38}$ or contrast medium. ${ }^{27}$ The US machines are portable and cheaper than the CT and the MRI scanners. The US is an essential modality for evaluating structural changes of the salivary glands. ${ }^{30,36}$ In order to evaluate salivary glands, a high parameter US equipment is required, comprising of a high electronic linear transducer of length approximately $4 \mathrm{~cm}$ long with a frequency of at least 7 megahertz $(7 \mathrm{MHz}){ }^{30}$

The US plays a significant role as a first line modality for initial diagnosis of patients presenting with the submandibular salivary glands swelling. The high resolution with Doppler technique provides excellent tissue characterization. ${ }^{39}$ Furthermore, it allows examination of the parenchymal blood flow and the glandular tissues vascular system. ${ }^{30}$ In addition to its ability to evaluate the parenchyma, the US can be used to examine the ducts revealing the ductal dilation. ${ }^{31}$ The US is a non-invasive modality which is affordable to many patients. ${ }^{31,32,34}$

A normal parotid salivary gland will display a homogenous speckle pattern structure. Any changes in the morphological structure of the salivary glands can be well depicted by an
US scan. Current literature shows that the US modality is gaining prominence as an assessment technique for salivary gland post radiation therapy. ${ }^{36,37}$ Cheng et al. ${ }^{36}$, reported on a study in which high resolution US was used to compare images of normal and post radiation parotid salivary glands. In that study it was established that grey scale US can be used successfully to access the size, echogenicity and the internal architecture of the parotid salivary glands. They described post radiation salivary glands as heterogeneous in structure, hyperechoic relative to adjacent muscles, with multiple lines or spots and hypoechoic. However, US cannot be used to successfully visualize the deep parotid gland because of the obstruction by the bony ramus. ${ }^{31}$

\section{Magnetic Resonance Sialography}

The Magnetic Resonance -Sialography (MR-Sialography) is a non-invasive 3 dimensional (3D) imaging technique. It relies on imaging static or slow flowing fluids in the body such as saliva. ${ }^{34,40}$ These fluids are imaged as high signal intensity bright structures against a dark background. MRSialography has a very low contrast. It shows slight changes in the salivary gland ducts. ${ }^{40}$

MR-Sialography is fast becoming a method of choice for evaluating radiation induced changes in the salivary glands ducts. When imaging the salivary gland ducts, saliva acts as a contrast medium. ${ }^{27,34,40}$ The modality acquires weighted images that are used to evaluate the degree of obstruction of the salivary ducts. It evaluates the ductal system of the two major pairs of salivary glands simultaneously. Saliva is seen as a hyper intense signal whilst the surrounding salivary gland tissues will appear hypointense. ${ }^{36}$

MR-Sialography modality has a potential to detect reduction in visibility of salivary glands ducts that have been subjected to radiation as low as 20Gy. Furthermore, it has the capacity to detect changes due to recovery mechanism on the salivary glands ducts. The changes are seen through improved visibility of the ducts, this makes MR-Sialography a promising modality for investigating radiation induced xerostomia. ${ }^{36}$ However, when compared to conventional sialography, it does not need an experienced operator. ${ }^{27}$ However, MR-Sialography cannot be used if saliva production is limited.

\section{Salivary glands Scintigraphy}

Salivary glands Scintigraphy is a non-invasive nuclear medicine technique that provides functional information. ${ }^{27}$ It has the ability to detect slight changes in the function of the salivary glands after parenchymal injury resulting from irradiation of the salivary glands during external beam 
therapy of head and neck tumours. ${ }^{41}$ The modality allows differentiation of osseous structures from soft tissues without burdening the patient with excessive radiation as is the case with $\mathrm{CT}{ }^{36}$

When studying the nature of the salivary gland tissue injury post-radiation therapy, technetium-99m pertechnetate $\left({ }^{99 \mathrm{~m}} \mathrm{Tc}\right)$ is injected intravenously to the patient. ${ }^{27,34,36}$ The ${ }^{99 m}$ Tc ions are then transported by the salivary glands epithelial cells. At the end of its biodistribution, the ${ }^{99 m} \mathrm{Tc}$ ions in the salivary glands emit gamma rays that can be imaged by the gamma camera. The acquired planar images are then quantified to give numerical values used to infer on the function of the salivary glands. ${ }^{36}$ The images may also be evaluated to both glandular uptake and excretory function.

The main advantage of salivary scintigraphy is that it is noninvasive and it is reproducible._It can be used to monitor recovery of salivary glands function. ${ }^{27}$ Furthermore; it can evaluate the two major pairs of the salivary glands single injected dose of ${ }^{99 \mathrm{~m}} \mathrm{Tc}$. However, the disadvantage of the technique is that it requires examination of the glands pre and post therapies in order to compare the degree of injury inflicted. Furthermore, it burdens the patient with radiation.

\section{Conclusion}

Salivary gland imaging post-radiation therapy of head and neck tumours is crucial in management of xerostomia. Imaging may be done using high resolution modalities such as US, CT and MRI and salivary glands scintigraphy. However, the high resolution modalities can only show morphological changes whereas salivary gland scintigraphy offers functional information. Furthermore, salivary glands scintigraphy has the ability to detect slight changes in both parenchymal function and excretion function of each individual salivary gland at earliest possible moment after radiation injury whereas the anatomical imaging modalities will start to demonstrate changes only when structural changes become evident long after the start of functional loss. In the event, there arises the need to evaluate the damage inflicted on the salivary gland ductal system, MRSialography is the most suitable imaging modality.

\section{References}

1. Samir S, El-Adalany Mk, Hamed EE. Value of dynamic contrast enhanced magnetic resonance imaging in differentiation between post-treatment changes and recurring salivary glands tumours. Egypt J Radiol Nucl Med. 2016;47:477-486.
2. Head and Neck Cancer. Radiologylnfo.org. 2016;1-5. https://www.radiologyinfo.org/en/pdf/head-neckcancer.pdf [Reviewed: Jul-21-2016].

3. Sood AJ, Fox NF, O'Connel PB, et al. Salivary gland transfer to prevent radiation-induced xerostomia: $A$ systematic review and meta-analysis. Oral Oncol. 2014;77-83.

4. D'cruz A, Lin T, Anand AK. Consensus recommendations for management of head and neck cancer in Asian countries: A review of international guidelines. Oral Oncol. 2013;49:872-877.

5. Lovelace TL, Fox NF, Sood AJ, et al. Management of radiotherapy-induced salivary hypofunction and consequent xerostomia in patients with oral and head and neck cancer: meta-analysis and literature review. Surg Oral Pathol Oral Radiol. 2014;117:595-607.

6. National Cancer Institute. A snap shot of head and neck cancers. [Internet]. 2012 [cited 2012 May 15]. Available from:

http://www.cancer.gov/researchandfunding/snapshots/ pdf/HeadandNeck-Snapshot.pdf

7. Lilly-Tariah O, Somefun AO, Ademo WL. Current evidence on the burden of head and neck cancers in Nigeria. Head and Neck Oncol. 2009;1:14.

8. Anwar W, Boulus DNK. Cancer registries in Africa: An emerging need. J Afr Cancer. 2012;4:140-141.

9. Gathere S, Mutuma G, Korir A, et al. Head and neck cancers four year trend at the Nairobi Cancer Registry. Afr J Health Sci. 2011;19:30-35.

10. Fehrenbach MJ, Herring SW. Anatomy of the head and neck. St Louis: Saunders Elsevier; 2007.

11. Chadi MJ, Georges GS, Albert F, et al. Major salivary gland aplasia and hypoplasia Down Syndrome: Review of literature and report of a case study. Clin Case Rep. 2017;5(6):939-944.

12. Fehrenbach MJ, Herring SW. Anatomy of the head and neck. St Louis: Saunders Elsevier; 2007.

13. Tucker AS. Salivary gland development. Sem Cell Biol. 2007;18:237-244.

14. Som PM, Brandwe MS. Salivary Glands: Anatomy and Pathology. Head and Neck Imaging. St Louis: Mosby; 2003.

15. Beale T, Madani G. Anatomy of the salivary glands. Semin Ultrasound CT MR. 2006;2:436-439.

16. Myers EN, Ferris RL. Salivary glands disorders. Berlin: Springer Heidelberg; 2007.

17. Humphrey SP, Williamson RT. A review of saliva: Normal composition, flow, and function. J Prosthet Dent. 2001;85:169-179.

18. Amerogengen AVN, Veerman ECl. Saliva- the defender of oral cavity. Oral Dis. 2002;8:12-22. 
19. Carmen LP. The role of saliva in maintaining oral health and as aid to diagnosis. Med Oral Pastol Cir Bucal. 2006;11:E449-E455.

20. De Souza Tolentio, et al. Oral adverse effects of head and neck radiotherapy: a literature review and suggestion of a clinical oral care guideline for irradiated patients. J. Appl. Oral Sci. 2011;19(5). http://dx.doi.org/10.1590/S1678-77572011000500003.

21. Dirix P, Nuyts S, Van den Bogaert. Radiation-induced xerostomia in patients with head and neck tumours. Cancer. 2006;107(11):2525-2527.

22. Atri R, Dhankhar R, Nair V, et al. Management of radiation induced xerostomia in head and neck cancers. J Oral Health Comm Dent. 2007;1:33-39.

23. Chambers MS, Garden AS, Kies MS, et al. Radiationinduced xerostomia in patients with head and neck cancer: pathogenesis, impact on quality of life, and management. Head Neck. 2004;26(9):796-807.

24. Jimenez-Heffernan A, Gomez M, Sanchez De Mora E, et al. Quantitative salivary gland scintigraphy in the head and neck cancer patients following radiotherapy. Rev Esp Med Nucl. 2010;29:165-171.

25. McGraw-Hill Dictionary of Scientific \& Technical Terms. [Internet] 2010. The McGraw-Hill Companies, Inc. Available from: http://encyclopedia2.thefreedictionary.com/Medical+im aging.

26. Afzelius $P$, Nielsen MY, Ewertsen $C$, et al. Imaging of the major salivary glands. Clin Physiol Funct Imaging. 2016 ;36(1):1-10. doi: 10.1111/cpf.12199.

27. Gopal S, Anusha VA. Salivary gland Imaging-an overview. Int J Inf Res Rev. 2017;4(09):4481-4485.

28. Agarwal AK, Kanekar SG, Imaging of submandibular and sublingual salivary glands. Neuroimag Clin N Am. 2018;28:227-243.

29. Chang JL. Salivary gland imaging. Gland-Preserving Salivary Surgery. 2017;15-26.

30. Zajkowiski P, Ochal-Choinnska A. Standards of assessment of salivary glands- An update. J Ultrason. 2016;16(65):175-190.

31. Yadav K, Pai A. Salivary glands imaging: Visualization of the oral lubricator. Mod App Dent Oral Health. 2018;1(4). MADOC MS.ID.000118.

32. Government of Western Australia. Salivary Glands Swelling. Diagnostic Pathways. Available on: www.imagingpathways.health.wa.gove.au.

33. Liu Y, Li J, Tan Y, et al. Accuracy of diagnosis of salivary gland tumours with the use of ultrasonography, computed tomography, and magnetic, resonance imaging: a meta-analysis. Oral Surg Oral Med Oral Pathol Oral Radiol. 2015;119(2):238-245.
34. Taneja A, Sachdev IS, Sethi S, et al. Salivry gland imaging: A pictorial essay. IJMDS. 2015;4(1):652-700.

35. McKie S, Brittenden J. Basic science: magnetic resonance imaging. Curr Orthopaed. 2005;19:13-19.

36. Cheng SC, WU VWC, Kwong DLW, et al. Assessment of post-radiotherapy salivary gland. Brit J Radiol. 2011;84:393-402.

37. Burke CJ, Thomas RH, Howlett D. Imaging of salivary glands. Br J Oral Maxillofac Surg. 2011;49:261-269.

38. Chan V, Perlas A. Basics of Ultrasound Imaging. Toronto: Springer Science + Business Media; 2011.

39. Freeling N, Crippa F, Marodi R. Staging and follow -up of traditional versus functional imaging approaches. 2016;60:157-166

40. Morimoto $\mathrm{Y}$, Ono K, Tanaka $\mathrm{T}$, et al. The functional evaluation of salivary glands using dynamic MR Sialography following citric acid stimulation: A preliminary study. Oral Surg Oral Med Oral Path Oral Radiol Endod. 2005;100:37-64.

41. Jimenez-Heffernan A, Gomez M, Sanchez De Mora E, et al. Quantitative salivary gland scintigraphy in the head and neck cancer patients following radiotherapy. Rev Esp Med Nucl. 2010;29:165-171.

\section{PEER REVIEW}

Not commissioned. Externally peer reviewed.

\section{CONFLICTS OF INTEREST}

None

\section{FUNDING}

None

\section{ETHICS COMMITTEE APPROVAL}

The study was approved by Medunsa Research Ethics Committee under the number MREC/P/280/2012: PG 
Figure 1: Flow chart showing the criteria used to select and exclude studies for the systematic review
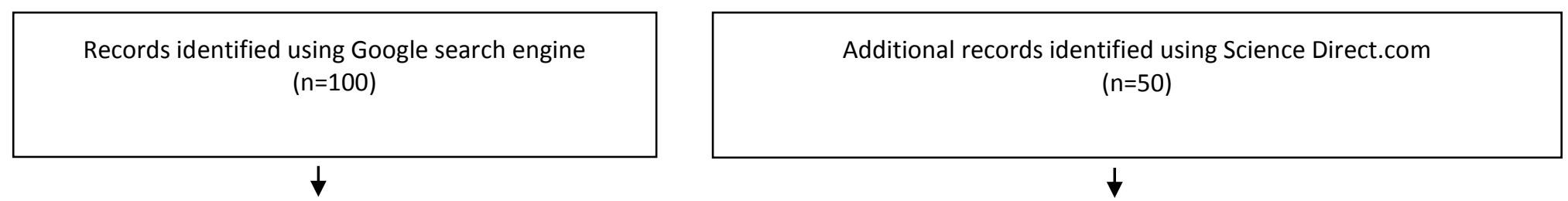

\begin{tabular}{|c|}
\hline Records after duplicates removed \\
$(\mathrm{n}=92)$
\end{tabular}

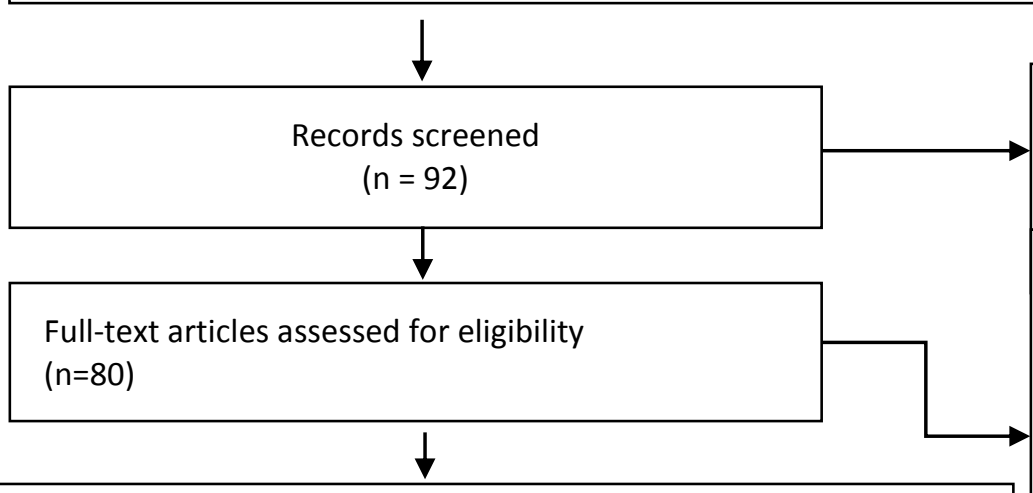

Records excluded

$$
(n=12)
$$

- Articles requiring payment

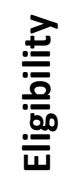

$\frac{\text { 잉 }}{\frac{0}{2}}$

Number of articles meeting the inclusion criteria: $(n=41)$

The selected articles contained information on:

- Salivary glands,

- Salivary glands imaging modalities post external beam radiation therapy for head and neck cancers

- Measurement of the degree ductal obstruction post external beam radiation treatment of head and neck tumours.

- Head and neck tumours

- Side effects radiation therapy of head and neck tumours. 